Check for updates

Cite this: RSC Adv., 2018, 8, 21768

Received 5th May 2018

Accepted 6th June 2018

DOI: $10.1039 / \mathrm{c} 8 \mathrm{ra03859g}$

rsc.li/rsc-advances

\section{Cellulose triacetate synthesis via one-pot organocatalytic transesterification and delignification of pretreated bagasse $\uparrow$}

\author{
Shiori Suzuki, ${ }^{a}$ Yoshiki Shibata, ${ }^{a}$ Daisuke Hirose, ${ }^{a}$ Takatsugu Endo, ${ }^{b}$ \\ Kazuaki Ninomiya, ${ }^{c}$ Ryohei Kakuchi (D) ${ }^{* d}$ and Kenji Takahashi (D) *a
}

Cellulose triacetate was synthesised by the transesterification reaction of mild acid-pretreated lignocellulosic biomass with a stable acetylating reagent (isopropenyl acetate, IPA) in an ionic liquid (1ethyl-3-methylimidazolium acetate, EmimOAc) which enabled the dissolution of lignocellulose as well as the organocatalytic reaction. The homogeneous acetylation of pretreated sugar-cane bagasse was carried out under mild conditions $\left(80^{\circ} \mathrm{C}, 30 \mathrm{~min}\right)$, and the subsequent reprecipitation processes led to enriched cellulose triacetate with a high degree of substitution (DS; 2.98$)$ and glucose purity ( 90\%) along with production of lignin acetate.

\section{Introduction}

Cellulose-based materials (e.g. cellulose acetate) have been widely utilised for diverse applications ${ }^{1-3}$ such as membrane matrices, ${ }^{4}$ optical films, ${ }^{5}$ cigarette filters, ${ }^{6}$ etc. ${ }^{7-11}$ Their importance can also be acknowledged from the viewpoint of green and sustainable chemistry. ${ }^{12}$ Cellulose is a major component of lignocellulosic biomass, so-called lignocellulose, that is independent from the instinctive competition with human food supplies. Despite the inherent advantage of cellulose-based materials, their synthesis from the original lignocellulose typically involves multi-step transformations that require harsh conditions. This is primarily attributable to the insolubility of cellulose, which leads to a fairly high cost associated with the purification step as compared to the processing of petroleumbased chemicals. ${ }^{13}$ To be precise, although cellulose comprises water-soluble glucose as a repeating unit, it is nearly

${ }^{a}$ Faculty of Natural System, Institute of Science and Engineering, Kanazawa University, Kakuma-machi, Kanazawa 920-1192, Ishikawa, Japan. E-mail: ktkenji@staff. kanazawa-u.ac.jp

${ }^{b}$ Department of Molecular Chemistry and Biochemistry, Faculty of Science and Engineering, Doshisha University, 1-3 Tatara Miyakodani, Kyoutanabe 610-0394, Kyoto, Japan

'Institute for Frontier Science Initiative, Kanazawa University, Kakuma-machi, Kanazawa 920-1192, Ishikawa, Japan

${ }^{d}$ Division of Molecular Science, Graduate School of Science and Technology, Gunma University, 1-5-1 Tenjin-cho, Kiryu 376-8515, Gunma, Japan. E-mail: kakuchi@ gunma-u.ac.jp

$\dagger$ Electronic supplementary information (ESI) available: Preparation methods of lignin samples, synthetic protocol of cellulose acetate, xylan acetate, and lignin acetate, ${ }^{13} \mathrm{C}$ NMR spectra, HPLC measurements for composition analysis, SEC measurement, determination of DS for the acetylated polysaccharides, quantitative ${ }^{31} \mathrm{P}$ NMR for determination of $\mathrm{OH}$ content of lignin samples. See DOI: $10.1039 / \mathrm{c} 8 \mathrm{ra} 03859 \mathrm{~g}$ insoluble in both common organic and aqueous solvents because of the extraordinarily strong hydrogen bonding networks within its polymeric structures. It is also worth noting that cellulose generally needs to be isolated from lignocellulose containing hemicellulose and lignin $^{14}$ prior to chemical modifications. The series of steps involved in this isolation process is known as pulping. The removal of lignin from lignocellulose, also called delignification, ${ }^{15}$ is one of the difficult tasks in the pulping system because of the low reactivity of lignin and the partial chemical linkages between the lignin and carbohydrates. ${ }^{16,17}$ Delignification is a multi-step process and requires a highly basic and oxidative medium at high temperatures. ${ }^{18,19}$ Furthermore, it suffers from poor selectivity and further purification by bleaching techniques can result in significant degradation of the constituent carbohydrates. ${ }^{20}$ Such multiple harsh delignification processes increase the cost and energy consumption in the entire cellulose purification process when compared to petroleum-based chemicals. Therefore, the production of highly pure cellulose-based materials from lignocellulose with simple and mild chemical treatments is in great demand to further supplement their inherent green chemistry attributes.

In recent years, ionic liquids (ILs) have emerged as a novel platform with an excellent dissolution ability ${ }^{21,22}$ for otherwise insoluble materials including cellulose. ${ }^{23,24}$ Using ILs as solvents for cellulose bypasses the conventional binary solvent mixtures (e.g. $N, N$-dimethylacetamide/LiCl) and designer solvents can be developed by tuning the structure of ILs..$^{25,26}$ Consequently, ILs have attracted significant attention for application in cellulose-related chemistry. In this context, we have previously reported that an ionic liquid, 1-ethyl-3methylimidazolium acetate (EmimOAc), acts not only as a cellulose solvent, but also as an organocatalyst in the 
transesterification reaction of cellulose. ${ }^{27-29}$ This bifunctional employment of EmimOAc allowed cellulose to react efficiently under mild conditions without environmentally hazardous catalysts (e.g. Brønsted acids such as sulfuric acid and organometallics) and additional activating reagents such as acid anhydrides and carboxylic halides.

It is worth noting that EmimOAc can dissolve both pure cellulose $^{30,31}$ and lignocellulose..$^{32,33}$ This foreknowledge encouraged us to investigate the applicability of our cellulose modification protocol ${ }^{27}$ toward lignocellulose. Chen et al. have also utilised this attribute of EmimOAc to successfully obtain acylated bagasse. ${ }^{34}$ Although these authors demonstrated the direct conversion of lignocellulose, the isolation of cellulose derivatives and the loss of the lignin fraction was not addressed in detail. Therefore, it is still a challenging task to enrich the target cellulose derivatives directly from lignocellulose under simple and mild conditions.

In this study, the direct acetylation of bagasse was conducted using the bifunctional IL, EmimOAc, and the subsequent reprecipitation in methanol $(\mathrm{MeOH})$ to separate both the polysaccharide and lignin acetyl derivatives was performed. In addition, we have proposed another synthetic process that allows enriching the cellulose derivatives from bagasse via a mild acid-pretreatment step, as depicted in Scheme 1. This involves (1) the selective acid hydrolysis of hemicellulose in bagasse, and (2) homogeneous acetylation of the pretreated bagasse and the subsequent reprecipitation process. This simple two-step process successfully afforded both enriched cellulose triacetate and lignin acetate.

\section{Experimental}

\subsection{General information}

2.1.1. Materials. 1-Ethyl-3-methyl-imidazolium acetate (EmimOAc) was obtained from Kanto Chemical Co., Inc. (Tokyo, Japan) and used without further purification. Isopropenyl acetate (IPA) and anhydrous dimethyl sulfoxide (DMSO) were obtained from Sigma-Aldrich Co., LLC. (St. Louis, MO, USA) and used as received. Bagasse was purchased from

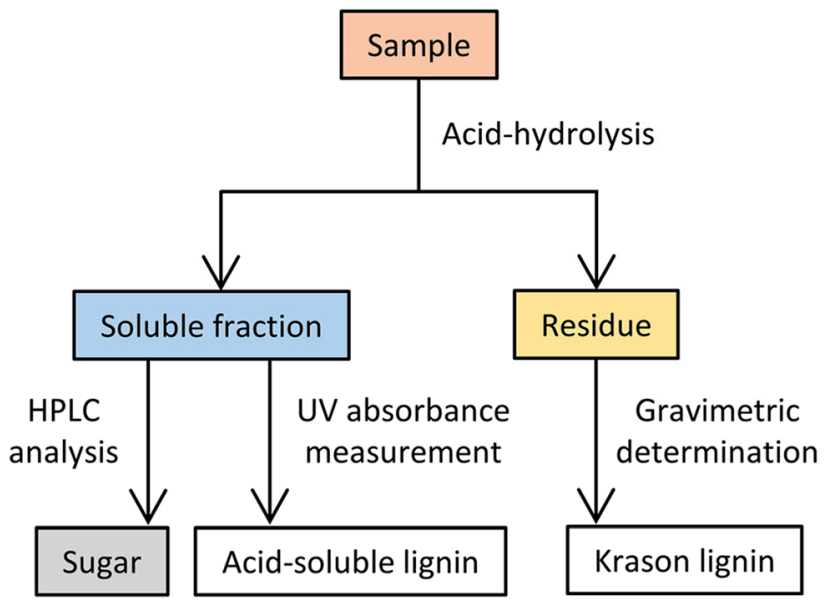

Scheme 2 Flow chart of the composition analysis of lignocellulosic material according to the NREL analytical method. ${ }^{42}$

the Toyota Motor Co. (Miyoshi, Japan) and cellulose (Avicel® $\mathrm{PH}-101$, particle size $<50 \mu \mathrm{m}$ ) was procured from Sigma-Aldrich Co., LLC. (St. Louis, MO, USA). The number-average degree of polymerization of Avicel was calculated to be $105 .{ }^{29}$ Xylan (from beech wood) was obtained from Tokyo Chemical Industry Co., Ltd. (Tokyo, Japan). Lignin was isolated from bagasse via enzymatic saccharification (see ESI $\dagger$ for the preparation of the lignin). All of the starting materials containing bagasse, Avicel, xylan, and lignin were dried under vacuum at $70{ }^{\circ} \mathrm{C}$ until a constant weight before use. Cellulase (Cellic ${ }^{\circledR}$ CTec2), a complex blend of cellulase, hemicellulase, and $\beta$-glucosidase (batch number VCNI 0008, 106 filter paper units (FPU) per millilitre), was purchased from Novozymes Japan, Ltd. (Chiba, Japan). The FPU of the cellulase was determined according to the National Renewable Energy Laboratory (NREL) method. ${ }^{35}$ Other chemicals were commercially available and used as received unless otherwise stated.

2.1.2. Instruments. ${ }^{1} \mathrm{H}$ and ${ }^{13} \mathrm{C}$ nuclear magnetic resonance (NMR) spectra were recorded using a JNM-ECA 600 spectrometer (JEOL Ltd., Tokyo, Japan) in deuterated solvents, and the

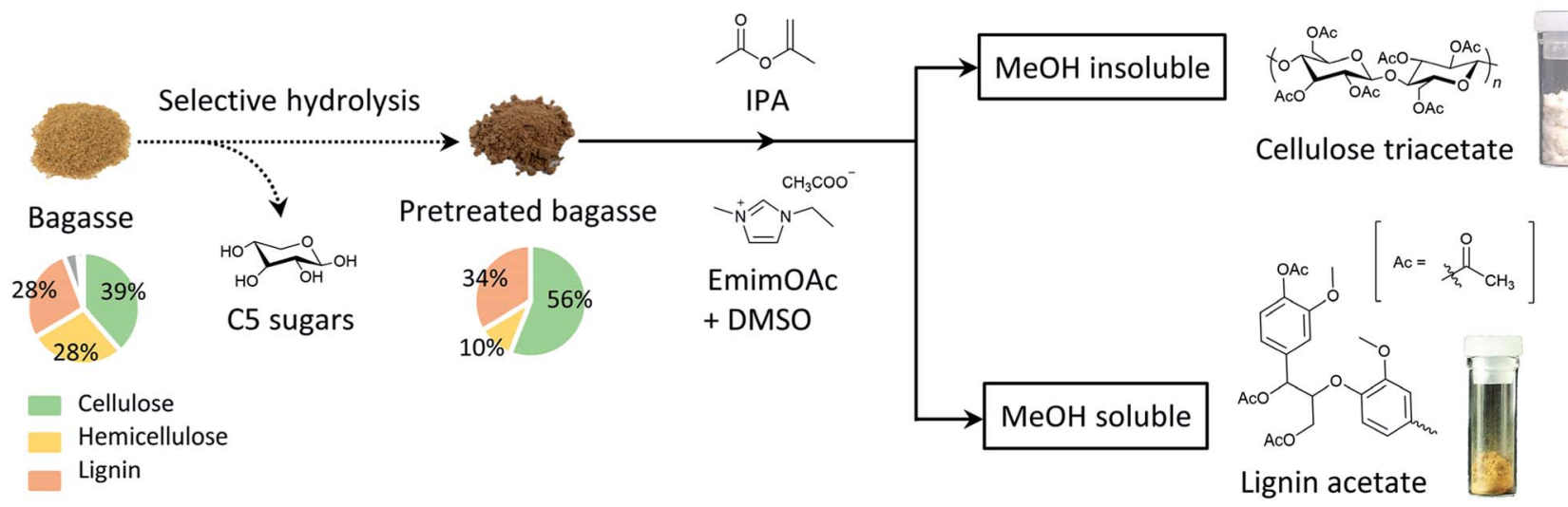

Scheme 1 Schematic representation of the organocatalytic transesterification of mild acid-pretreated bagasse and successive fractionation into enriched cellulose triacetate and lignin acetate by solid-liquid separation. 
Table 1 Isolated yields of acetylated products from bagasse

\begin{tabular}{lll}
\hline Fraction & Main component & Isolated yield ${ }^{a, b}(\%)$ \\
\hline MeOH-insoluble & Polysaccharide acetate & $48 \pm 3.8^{c}$ \\
MeOH-soluble & Lignin acetate & $72 \pm 2.1$
\end{tabular}

${ }^{a}$ Average yields $(n=3) .{ }^{b}$ Theoretical yields were calculated from the composition of bagasse based on the hypothesis that hemicellulose was only composed of xylan. ${ }^{c}$ Collected as a chloroform-soluble portion.

chemical shifts $(\delta)$ were given in ppm referenced to either the residual solvent peak or tetramethylsilane (TMS, $\delta=0$ ) as the internal standard. Fourier transform infrared spectrometer (FTIR) spectra were recorded on a Thermo Fisher Scientific Nicolet is10 (Thermo Fisher Scientific, Inc., Tokyo, Japan) spectrophotometer equipped with an attenuated total reflection (ATR) unit. All the centrifugation processes $\left(12500 \times g, 4{ }^{\circ} \mathrm{C}, 60 \mathrm{~min}\right)$ were conducted using a compact high-speed refrigerated centrifuge (KUBOTA 6930, Kubota Co., Ltd., Osaka, Japan). The molecular weights of the polymeric samples were determined by size exclusion chromatography (SEC, Prominence UFLC system, Shimadzu Co., Kyoto, Japan) based on polystyrene standards. All SEC measurements were carried out at $40{ }^{\circ} \mathrm{C}$ using TSK gel $\alpha$ $\mathrm{M}$ (Tosoh Co., Tokyo, Japan), and $0.01 \mathrm{~mol} \mathrm{~L}^{-1} \mathrm{LiBr}$ in dimethylformamide (DMF, HPLC grade, Kanto Chemicals Co., Inc., Tokyo, Japan) was used as an eluent at the flow rate of 1.0 $\mathrm{mL} \min ^{-1}$. The high-performance liquid chromatograph

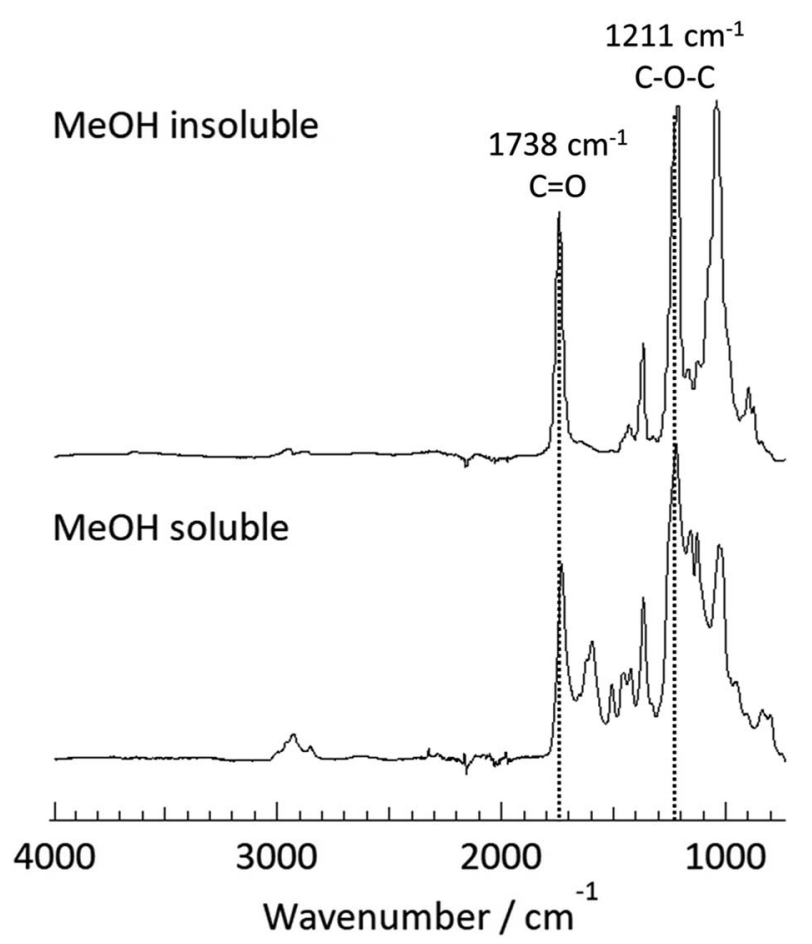

Fig. 1 ATR-mode FT-IR spectra of the $\mathrm{MeOH}$-insoluble fraction (upper) and the $\mathrm{MeOH}$-soluble fraction (lower) isolated after the direct acetylation of bagasse at $80^{\circ} \mathrm{C}$ for $30 \mathrm{~min}$.

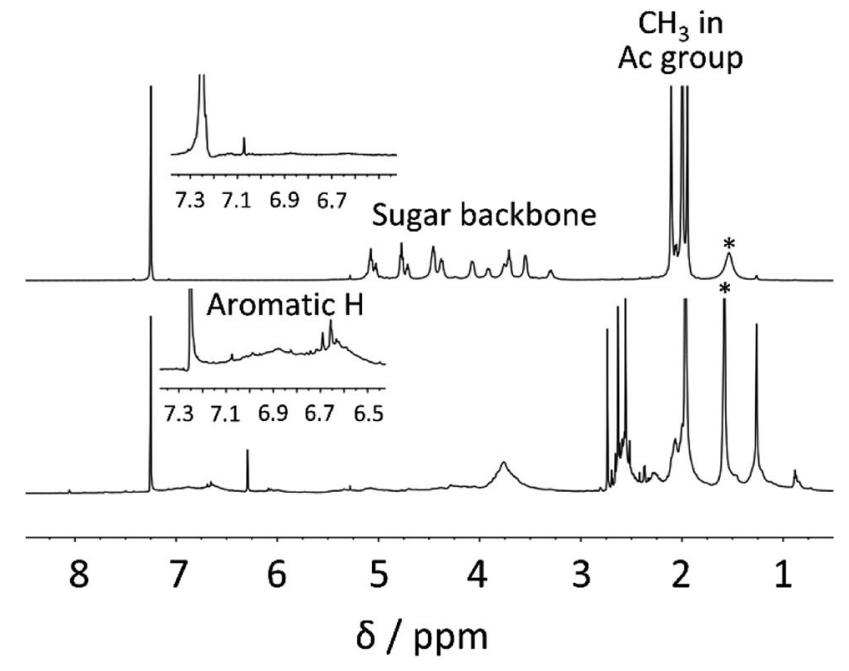

Fig. $2{ }^{1} \mathrm{H}$ NMR spectra of the $\mathrm{MeOH}$-insoluble fraction (upper) and the $\mathrm{MeOH}$-soluble fraction (lower) isolated after the direct acetylation of bagasse at $80{ }^{\circ} \mathrm{C}$ for $30 \mathrm{~min}$. Both ${ }^{1} \mathrm{H}$ NMR spectra were measured in $\mathrm{CDCl}_{3}$ at $55^{\circ} \mathrm{C}$.

(HPLC) was equipped with a refractive index (RI) detector (Shimadzu Co., Kyoto, Japan) and a CARBOSep CHO-682 column (Tokyo Chemical Industry Co., Ltd., Tokyo, Japan). HPLC measurements were conducted at $85{ }^{\circ} \mathrm{C}$ with an ultrapure water mobile phase and flow rate of $0.4 \mathrm{~mL} \mathrm{~min}^{-1}$. A DU® series 700 UV/VIS scanning spectrophotometer (Beckman Coulter, Inc, Tokyo, Japan) was used for UV measurements at $240 \mathrm{~nm}$ with a quartz cell (path length: $1 \mathrm{~cm}$ ).

\subsection{Synthesis and isolation protocols}

2.2.1. Direct acetylation of bagasse. Initially, bagasse was crushed using a grinder mill (Y-308B, Osaka Chemical Co., Ltd., Osaka, Japan) into a powder having particles of size smaller than $250 \mu \mathrm{m}$. Prior to use, the collected powder was further purified by Soxhlet extraction using dichloromethane for at least $16 \mathrm{~h}$, and then drying in a vacuum oven at $70{ }^{\circ} \mathrm{C}$ for $24 \mathrm{~h}$. The purified bagasse $(600 \mathrm{mg}, 6 \mathrm{wt} \% / \mathrm{EmimOAc})$ was transferred to a Schlenk tube with $10 \mathrm{~g}$ of EmimOAc, and the mixture was dried under vacuum at $80{ }^{\circ} \mathrm{C}$ for $2 \mathrm{~h}$. After argon gas was flushed into the reaction mixture, DMSO $(15 \mathrm{~mL})$ was added to the solution as a co-solvent to assist in the dissolution of bagasse. ${ }^{36,37} \$$ The obtained solution was stirred at $110{ }^{\circ} \mathrm{C}$ for $16 \mathrm{~h}$ to obtain a black-brown homogeneous viscous solution. After cooling the solution to $80^{\circ} \mathrm{C}$, an excess amount of IPA $(20 \mathrm{~mL})$ was added to the solution, and acetylation was conducted by stirring for $30 \mathrm{~min}$. Subsequently, the highly viscous reaction mixture was diluted with a small amount of dichloromethane and poured into $\mathrm{MeOH}(600 \mathrm{~mL})$. The precipitated polymer was filtered and washed with $\mathrm{MeOH}$ to yield a pale yellowish gel-like solid. After the $\mathrm{MeOH}$-insoluble gel was dissolved in chloroform $(300 \mathrm{~mL})$ by stirring at room temperature for $1 \mathrm{~h}$, the resultant

† Since all the procedures were handled under either vacuum or Ar atmosphere, the water concentration of the reaction mixture was practically negligible. 

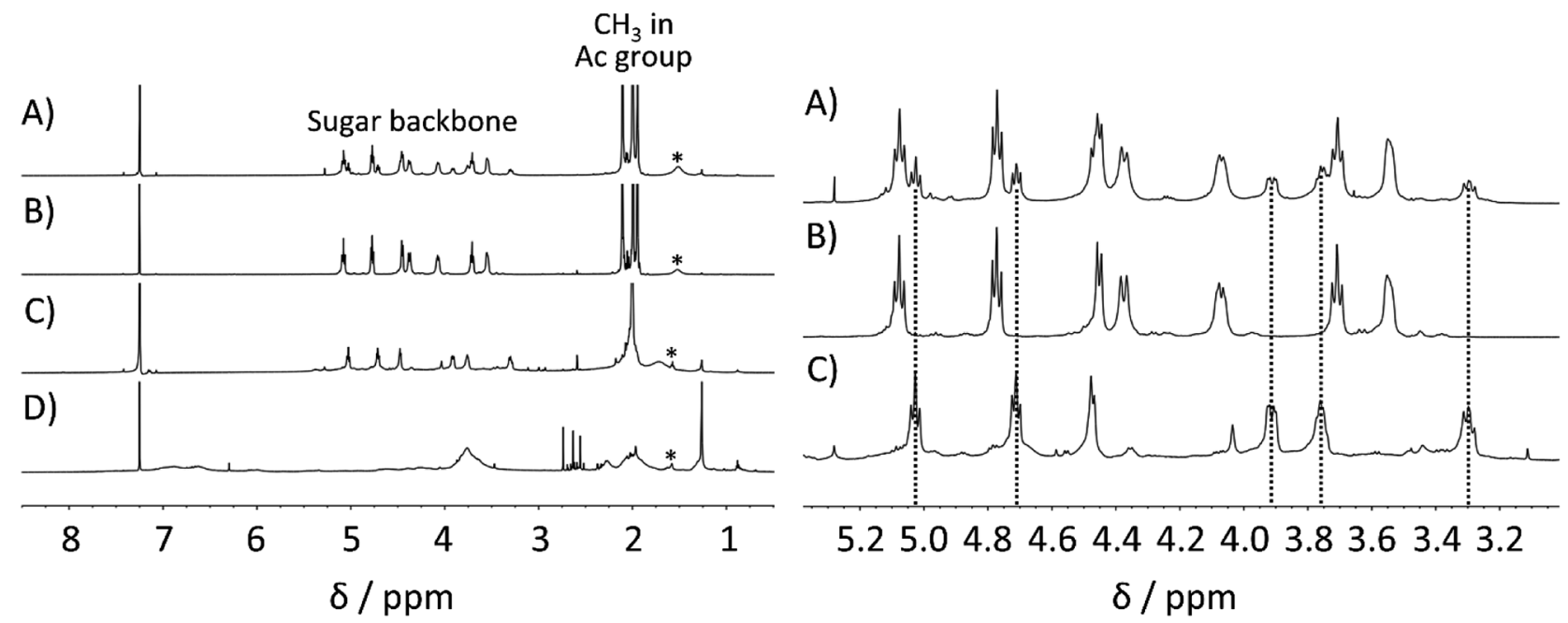

Fig. $3{ }^{1} \mathrm{H}$ NMR spectra in $\mathrm{CDCl}_{3}$ at $55^{\circ} \mathrm{C}$ (left) and the expansion (right) of (A) polysaccharide acetate in the MeOH-insoluble fraction after the direct acetylation of bagasse at $80^{\circ} \mathrm{C}$ for $30 \mathrm{~min}$, (B) cellulose acetate, (C) xylan acetate, and (D) lignin acetate as reference samples.

solution was filtered to remove the chloroform-insoluble residue. The average yield $(n=2)$ of the residue was $192 \mathrm{mg}$. The chloroform filtrate was then concentrated and reprecipitated into $\mathrm{MeOH}(300 \mathrm{~mL})$. The pale yellowish gel-like residue was subsequently washed with $\mathrm{MeOH}$ and distilled water. After freeze drying for two days, polysaccharide acetate was obtained. The average yield ( $n=3$ ) was $326 \mathrm{mg}$.

The $\mathrm{MeOH}$ filtrate obtained in the first precipitation step was concentrated using an evaporator at $40{ }^{\circ} \mathrm{C}$, and a black viscous liquor containing EmimOAc, DMSO, and lignin derivatives was obtained. Acetone $(100 \mathrm{~mL})$ was added to the obtained solution to produce a clear homogeneous mixture. To remove $\mathrm{Emim}^{+}$, the homogeneous acetone solution was stirred at room temperature for $2 \mathrm{~h}$ along with a strong-acidic cation exchange resin (50 g, Amberlite ${ }^{\circ}$ IRN-77, $\mathrm{H}^{+}$form, Sigma-Aldrich Co., LLC., St. Louis, MO, USA). The used resin was removed by filtration and washed with acetone. The acetone filtrate was again evaporated at $40{ }^{\circ} \mathrm{C}$, and the resultant mixture was added dropwise to distilled water $(600 \mathrm{~mL})$ to precipitate light brown coloured particles. After centrifugation, the residue was washed with distilled water and freeze dried for two days to obtain lignin acetate. The average yield $(n=3)$ was $144 \mathrm{mg}$.

2.2.2. Acetylation of mild acid-pretreated bagasse. To selectively remove hemicellulose as C5 sugars, a mild acidpretreatment of the bagasse was conducted by following the literature procedure. ${ }^{38}$

The purified bagasse powder $(3.0 \mathrm{~g})$ was presoaked in a $3.0 \mathrm{~mol} \mathrm{~L}^{-1}$ sulfuric acid aqueous solution with a $3 \%(\mathrm{w} / \mathrm{w})$ total solids loading at room temperature for $4 \mathrm{~h}$. This slurry, which was prepared in a $250 \mathrm{~mL}$ polytetrafluoroethylene (PTFE) inner tube embedded within a pressure resistant stainless-steel vessel, was heated in a rotary type oven (RDV-TM2, SAN-AI Kagaku Co., Ltd., Aichi, Japan) at $160{ }^{\circ} \mathrm{C}$ for $20 \mathrm{~min}\left(160{ }^{\circ} \mathrm{C}\right.$ temperature was reached in $50 \mathrm{~min}$ from room temperature). After the reaction container was cooled to room temperature in an ice bath, the acidic slurry was vacuum filtered. The red- brown coloured residue was washed with distilled water (100 $\mathrm{mL}$ ) at least five times and dried in a vacuum oven at $70^{\circ} \mathrm{C}$ for $24 \mathrm{~h}$ to obtain pretreated bagasse that mainly contained cellulose and lignin.

The homogeneous acetylation process using the EmimOAc/ DMSO system and the subsequent separation of the synthesized cellulose triacetate in the $\mathrm{MeOH}$-insoluble fraction and lignin acetate in the $\mathrm{MeOH}$-soluble fraction were performed in the same manner for direct bagasse conversion as described in the experimental Section 2.2.1. The obtained cellulose triacetate was further purified by chloroform dissolution and filtration. 9 The average yields ( $n=3$ ) were $423 \mathrm{mg}$ (cellulose triacetate) and $197 \mathrm{mg}$ (lignin acetate).

\subsection{Composition analysis}

Prior to composition analysis, deacetylation of the acetylated polysaccharides was conducted according to the literature procedure $^{39}$ because this analytical method was intended for the chemically-unmodified lignocellulose.

Polysaccharide acetate from bagasse, cellulose triacetate from pretreated bagasse, and cellulose acetate from Avicel were dried under vacuum at $70{ }^{\circ} \mathrm{C}$ for $24 \mathrm{~h}$ before use. The acetylated polysaccharide $(200 \mathrm{mg}$ ) was weighed into a $30 \mathrm{~mL}$ vial. A mixed solvent $(20 \mathrm{~mL})$ of acetone/MeOH $(1: 1, \mathrm{v} / \mathrm{v})$ was added and stirred at room temperature for $2 \mathrm{~h}$. Deacetylation was conducted by stirring the mixture at room temperature for $24 \mathrm{~h}$ with a $1.0 \mathrm{~mol} \mathrm{~L}^{-1}$ sodium hydroxide aqueous solution $(2.0 \mathrm{~mL})$. The resultant solution was filtered after centrifugation. The pale yellow powdery residue was washed repeatedly with distilled water and collected after freeze drying for two days. The average yields $(n=3)$ of the deacetylated products were $105 \mathrm{mg}$ (bagasse-derived polysaccharide acetate), $109 \mathrm{mg}$ (pretreated

I In the case of the acetylation of pretreated bagasse, only a small amount of the chloroform-insoluble material was obtained. 
bagasse-derived cellulose triacetate), and $116 \mathrm{mg}$ (Avicel-derived cellulose acetate).

The composition analyses of the deacetylated polysaccharides and the starting materials (i.e., bagasse and pretreated bagasse) were conducted according to the standard biomass analytical method provided by the NREL. ${ }^{40}$ A simplified flow chart of the composition analysis protocol is depicted in Scheme 2. This procedure essentially involves the following two steps: (1) acid hydrolysis of the sample to fractionate into two quantifiable components depending on its solubility in an acidic solution, and (2) quantification of the hydrolysate obtained in the first step via HPLC and UV measurements.

\section{Results and discussion}

\subsection{Direct acetylation of bagasse and subsequent fractionation into polysaccharide acetate and lignin acetate}

To synthesise cellulose derivatives with sufficient purity directly from lignocellulose, direct acetylation of bagasse was carried out. In this context, bagasse was subjected to a transesterification reaction in a mixed solvent of EmimOAc/DMSO, which enabled both lignocellulose dissolution and organocatalytic transesterification. Considering the common solubility behaviour of cellulose acetate, ${ }^{27,28}$ hemicellulose acetate, ${ }^{29}$ and lignin acetate, ${ }^{\mathbf{1 1}} \mathrm{MeOH}$ was selected as the anti-solvent for polysaccharide acetyl derivatives and as a good solvent for lignin acetate. $\S$ Additionally, chloroform was selected as good solvent for the obtained acetylated polysaccharide because it can solubilise cellulose acetate exceptionally well with a high degree of substitution (DS). Therefore, the obtained reaction mixture was precipitated into $\mathrm{MeOH}$ and then fractionated into $\mathrm{MeOH}$-insoluble and soluble portions. The $\mathrm{MeOH}$-insoluble portion containing the polysaccharide acetate was further purified by chloroform dissolution, and the isolated product yields are summarised in Table 1.

The bagasse sample $(600 \mathrm{mg})$ used in this study was revealed to contain $66 \mathrm{wt} \%$ polysaccharides corresponding to $398 \mathrm{mg}$ (see Table $\mathrm{S} 4$ in ESI $\uparrow$ for the composition of the starting materials). If all of the hydroxyl $(\mathrm{OH})$ groups of the polysaccharides were acetylated, $683 \mathrm{mg}$ of the polysaccharide acetate was expected to be generated, given that hemicellulose in the bagasse sample was essentially composed of xylan. Therefore, the collected weight of $326 \mathrm{mg}$ corresponded to $48 \%$ isolated yield. Along with the carbohydrates, the lignin component was $28 \mathrm{wt} \%$ of the employed bagasse as determined by the composition analysis (Table S4 in ESI $\dagger$ ). In addition, the alcoholic, phenolic, and carboxylic acid $\mathrm{OH}$ concentrations of lignin were separately estimated by quantitative ${ }^{31} \mathrm{P}$ NMR analysis, ${ }^{\mathbf{4 2 , 4 3}}$ which indicated that $4.53 \mathrm{mmol} \mathrm{g}^{-1}$ of total $\mathrm{OH}$ groups were present in bagasse-derived lignin (Tables S6-S7 in ESI $\dagger$ for the determination of the lignin $\mathrm{OH}$ concentration). Thus, $198 \mathrm{mg}$ of lignin acetate was theoretically expected to be produced from

$\S$ Among alcohol derivatives, $\mathrm{MeOH}$ was selected because of its low boiling point and low cost. In addition, other common organic solvents were not selected because acetone, for example, can partially dissolve polysaccharide acetate as well as lignin acetate.
Table 2 Isolated yield of acetylated products from pretreated bagasse

\begin{tabular}{lll}
\hline Fraction & Main component & Isolated yield ${ }^{a, b}(\%)$ \\
\hline MeOH-insoluble & Cellulose triacetate & $61 \pm 6.7^{c}$ \\
MeOH-soluble & Lignin acetate & $82 \pm 1.0$
\end{tabular}

${ }^{a}$ Average yields $(n=3) \cdot{ }^{b}$ Estimated on the basis of the results of the composition analyses of both pretreated bagasse and the acetylated products. ${ }^{c}$ Collected as the chloroform-soluble portion. The amount of the chloroform-insoluble portion was negligible in the case of pretreated bagasse.

bagasse via the acetylation process, thus providing a reasonable agreement with the experimental result of $144 \mathrm{mg}$ (corresponding to $72 \%$ isolated yield). Therefore, both polysaccharide acetate and lignin acetate were obtained from bagasse in acceptable to moderate isolated yields.

To provide clear chemical information, both fractions were characterized by FT-IR and ${ }^{1} \mathrm{H}$ NMR. In the FT-IR spectra (Fig. 1), a strong absorption at $\sim 1738 \mathrm{~cm}^{-1}$ corresponding to $\mathrm{C}=\mathrm{O}$ stretching was observed for both fractions. Furthermore, the band at $1211 \mathrm{~cm}^{-1}$ (C-O-C ether stretching) was also detected for both fractions. These observations implied that the transesterification reaction of bagasse and subsequent reprecipitation processes afforded the acetylated polymeric fractions depending on their solubility in $\mathrm{MeOH}$. A detailed insight into the chemical structures of these two fractions were provided by ${ }^{1} \mathrm{H}$ NMR analysis, as shown in Fig. 2. In the spectrum of the $\mathrm{MeOH}$-insoluble fraction (Fig. 2 (upper)), the chemical shifts corresponding to the acetyl protons were observed in the spectral range of 1.9-2.1 ppm. In addition, the signals in the range of 3.2-5.1 ppm corresponded to the sugar skeleton. As there were no clear peaks arising from the aromatic protons of lignin in the spectrum, it was concluded that the $\mathrm{MeOH}$ insoluble fraction was mainly composed of polysaccharide acetate.

In the same manner as that for the $\mathrm{MeOH}$-insoluble fraction, peaks in the range of 1.9-2.4 ppm arising from the acetyl groups were detected in the spectrum of the $\mathrm{MeOH}$-soluble fraction (Fig. 2 (lower)). The broad peaks in the spectral range of 6.5$7.2 \mathrm{ppm}$ corresponded to the aromatic skeleton of lignin. It is noteworthy that there were no clear peaks attributable to polysaccharides. These experimental data indicated that the isolation of polysaccharide acetate from lignin acetate could be accomplished by a simple reprecipitation process with $\mathrm{MeOH}$.

Next, a precise investigation of polysaccharide acetate was conducted to confirm the purity of the obtained polysaccharide acetate. Hence, the acetylated lignocellulosic components, namely, cellulose acetate, xylan acetate, and lignin acetate, were separately prepared as reference samples (see ESI $\dagger$ for the synthetic protocols). The ${ }^{1} \mathrm{H}$ NMR spectrum of bagasse-derived polysaccharide acetate was compared with those of the reference samples, as depicted in Fig. 3. As expected from the chemical composition of bagasse, the above-mentioned ${ }^{1} \mathrm{H}$ NMR spectrum was in a reasonable agreement with the superimposition of the spectra of cellulose acetate and that of xylan acetate without a distinct contribution from lignin acetate. In 


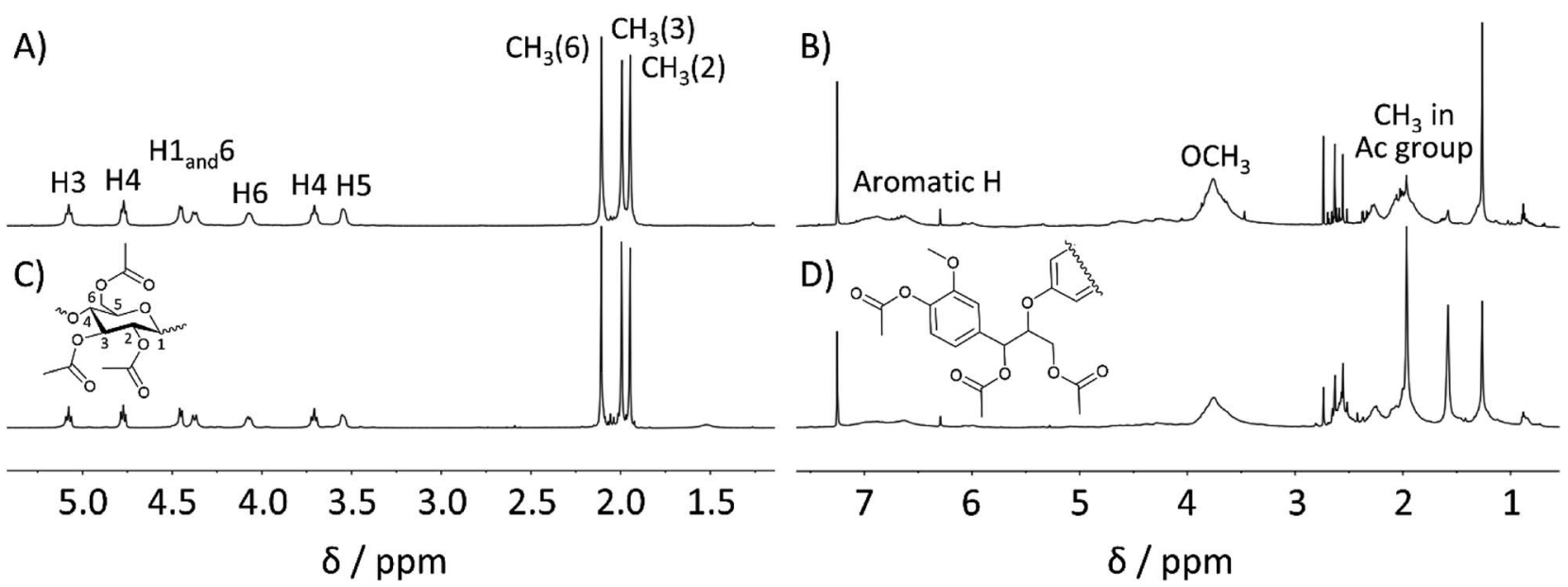

Fig. $4{ }^{1} \mathrm{H}$ NMR spectra in $\mathrm{CDCl}_{3}$ at $55^{\circ} \mathrm{C}$ of (A) cellulose triacetate in the $\mathrm{MeOH}$-insoluble fraction, (B) lignin acetate in the MeOH-soluble fraction, which were isolated after direct acetylation of pretreated bagasse at $80^{\circ} \mathrm{C}$ for 30 min (upper), (C) cellulose acetate synthesized from Avicel, and (D) lignin acetate sample synthesized from lignin isolated from bagasse as reference samples.

addition, the ${ }^{13} \mathrm{C}$ NMR spectra also showed that the bagassederived polysaccharide acetate was composed of not only cellulose, but also other polymeric sugars (Fig. S1 in ESI $\dagger$ ). These analyses demonstrated that the direct bagasse transesterification in EmimOAc/DMSO mixed system led to two fractions via a simple reprecipitation process, which corresponded to (1) the $\mathrm{MeOH}$-insoluble polysaccharide-rich fraction mainly comprising cellulose acetate and xylan acetate, and (2) the $\mathrm{MeOH}$-soluble fraction essentially containing lignin acetate.

\subsection{Acetylation of mild acid-pretreated bagasse and enrichment of cellulose triacetate}

As discussed in the previous section, the subsequent $\mathrm{MeOH}$ reprecipitation process after the direct modification of bagasse successfully afforded polysaccharide acetate without any harsh delignification treatments. Thus, the next challenge was to further separate cellulose acetate from hemicellulose acetate. The chemical structures of both cellulose and hemicellulose

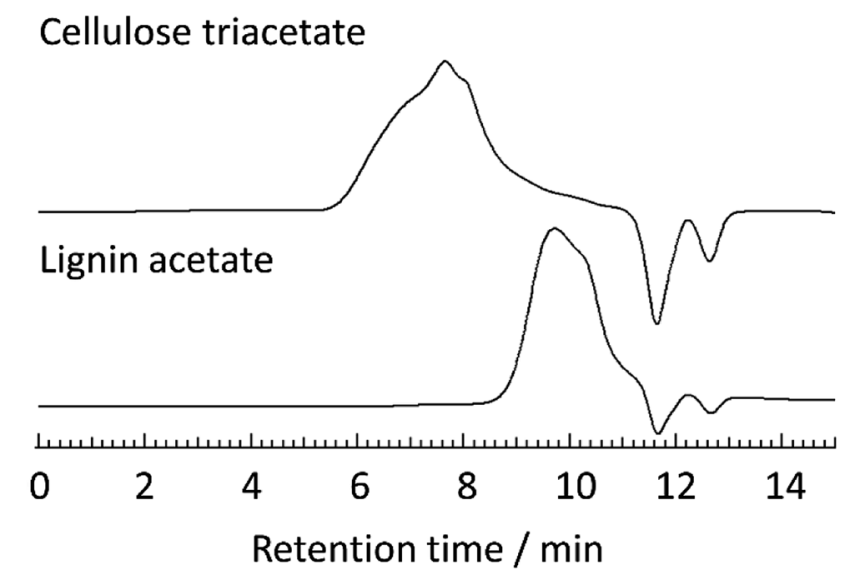

Fig. 5 SEC charts of cellulose triacetate (upper) and lignin acetate (lower) from pretreated bagasse measured at $40^{\circ} \mathrm{C}$ in DMF containing $0.01 \mathrm{~mol} \mathrm{~L}^{-1} \mathrm{LiBr}$. acetates are similar to each other and their solubility in common organic solvents is also similar in principle. It was expected that the similarity in properties would suppress the complete separation of cellulose acetate from hemicellulose acetate simply based on the solubility, as compared to the case of polysaccharide acetate and lignin acetate.

We then hypothesised that a mild acid-pretreatment of the lignocellulose could lead to further separation of the lignocellulosic components, namely, cellulose and hemicellulose, because the mild acid hydrolysis could selectively destroy hemicellulose in the lignocellulose while cellulose and lignin remained intact. ${ }^{38}$ In order to chemically prove our concept, the mild acid-pretreated bagasse was subjected to transesterification in the EmimOAc/DMSO system in a manner similar to the bagasse transesterification mentioned above.

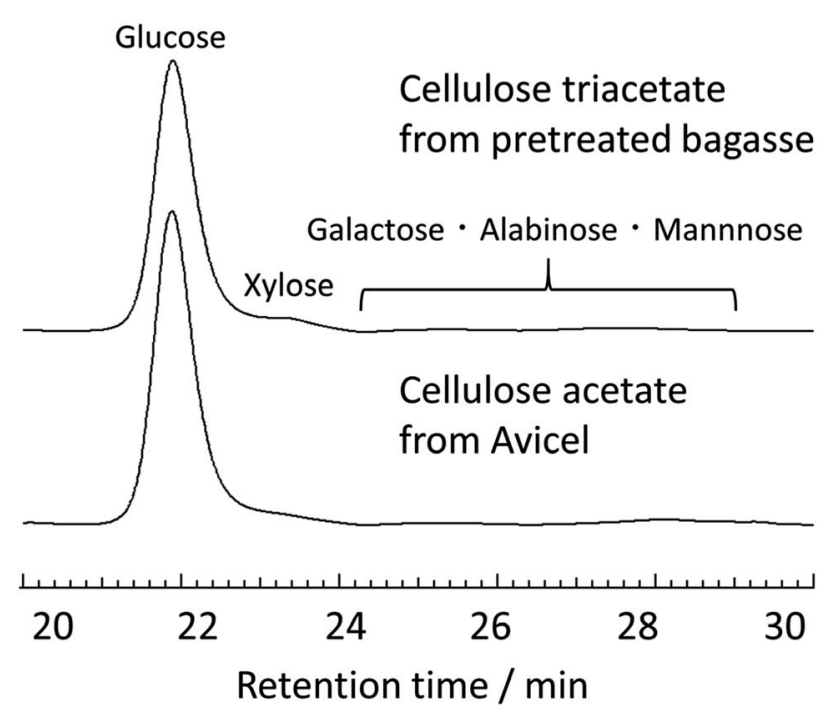

Fig. 6 HPLC chromatograms of the acid-hydrolysed mixtures from cellulose triacetate from the pretreated bagasse (upper) and cellulose acetate from Avicel (lower) measured at $85^{\circ} \mathrm{C}$ in ultra-pure water. 
Table 3 Compositions of cellulose triacetate from pretreated bagasse and cellulose acetate from Avicel as a reference sample

\begin{tabular}{|c|c|c|c|c|}
\hline Material & \multicolumn{4}{|c|}{ Composition (wt\%) } \\
\hline Cellulose acetate from Avicel ${ }^{b, c}$ & 55.9 & 1.4 & 2.2 & 40.3 \\
\hline
\end{tabular}

After the reaction, the EmimOAc/DMSO reaction medium was also fractionated into the $\mathrm{MeOH}$-insoluble and soluble portions. The isolated yields of the two fractions are summarised in Table 2 (the mass balance diagram is shown in Table S1 in $\left.\mathrm{ESI}_{\dagger}\right)$, which were reasonable agreement with the corresponding theoretical yields estimated by the composition of pretreated bagasse (Table S4 in ESI $\dagger$ ).

Furthermore, the ${ }^{1} \mathrm{H}$ NMR spectrum of the $\mathrm{MeOH}$-insoluble portion was in good agreement with that of cellulose acetate synthesized from Avicel (Fig. 4 (left)). Peaks corresponding to hemicellulose in the spectral range of 3.5-5.5 ppm and those attributable to lignin in the range of 6.2-7.2 ppm were not observed within the ${ }^{1} \mathrm{H}$ NMR sensitivity range. The ${ }^{13} \mathrm{C}$ NMR spectrum further confirmed that the $\mathrm{MeOH}$-insoluble portion was correctly assigned as cellulose acetate (Fig. S2 in ESI $\dagger$ ). In addition, the average DS $(n=3)$ of cellulose acetate obtained from pretreated bagasse was determined to be 2.98 , corresponding to $\sim 99 \% \mathrm{OH}$ group conversion (see ESI $\dagger$ for the DS determination). According to the US Federal Trade Commission, cellulose triacetate is defined as at least $92 \%$ of $\mathrm{OH}$ groups in the cellulose being acetylated. ${ }^{44}$ Thus, the cellulose acetate obtained from pretreated bagasse was assigned as cellulose triacetate.

On the other hand, the ${ }^{1} \mathrm{H}$ NMR spectrum of the $\mathrm{MeOH}-$ soluble portion (depicted in Fig. 4 (right)) was also in good agreement with that of lignin acetate synthesised from lignin that was isolated from bagasse without any distinct peaks corresponding to the polysaccharides. The $\mathrm{OH}$ group conversion of lignin in pretreated bagasse was estimated to be $77 \%$ based on the quantitative ${ }^{31} \mathrm{P}$ NMR measurements (Table S7 in ESI $\dagger$ ). These results demonstrated the efficient acetylation of both cellulose and lignin in pretreated bagasse using the EmimOAc/ DMSO system, and the subsequent reprecipitation processes enabled the synthesis of cellulose triacetate along with the production of lignin acetate. Considering the potential applications of lignin derivatives in diverse fields, ${ }^{\mathbf{1 9}, 45}$ this concurrent production of lignin acetate could be beneficial.

To further ensure the purity of the recovered cellulose triacetate, elemental analyses and SEC were measured. In addition, composition analyses by HPLC measurements were also performed for the monomeric sugars obtained after acid hydrolysis of the pretreated bagasse-derived cellulose triacetate. The elemental analysis of the cellulose triacetate was as follows: C, 49.19, H, 5.63, N, 0.20. This result was in good agreement with that of cellulose acetate obtained from Avicel (C, 49.18, $\mathrm{H}$, $5.56, \mathrm{~N}, 0.16$ ), demonstrating the easy purification method of cellulose triacetate from pretreated bagasse. Furthermore, SEC measurements were carried out for the cellulose triacetate and lignin acetate obtained from pretreated bagasse (Fig. 5, SEC charts and molecular weight $\left(M_{\mathrm{w}}\right)$ information of the other acetylated products are listed in ESI, Fig. S3-S4 and Table S2 $\dagger$ ). In general, cellulose acetate should be a linear polymer and form random coil conformations in the solution state. On the other hand, lignin acetate should have a branched structure and its hydrodynamic volume in solution is expected to be lower than that of cellulose acetate. As expected, the peak of cellulose triacetate obtained from pretreated bagasse did not overlap with that of lignin acetate (Fig. 5). The $M_{\mathrm{w}}$ of cellulose triacetate was $1.3 \times 10^{3} \mathrm{~kg} \mathrm{~mol}^{-1}$ and that of lignin acetate was determined to be $3.7 \mathrm{~kg} \mathrm{~mol}{ }^{-1}$. These observations showed that cellulose triacetate was sufficiently separated from lignin acetate, and the obtained cellulose triacetate maintained its polymeric structure throughout the mild acid pretreatment.

The monomeric structure of cellulose triacetate obtained from the pretreated bagasse was precisely determined by HPLC analysis. Specifically, the acid-hydrolysed polysaccharide samples were analysed by comparing with glucose, xylose, galactose, arabinose, and mannose as standard sugars (the details of composition analysis were summarized in ESI, please see Fig. S5-S6†). Fig. 6 shows the HPLC charts of the hydrolysates from cellulose triacetate that was obtained from both pretreated bagasse and Avicel and the determined compositions are summarised in Table 3. The contamination of hemicellulose $(\sim 2.1 \mathrm{wt} \%)$ and lignin $(\sim 5.2 \mathrm{wt} \%)$ was in the range observed for the Avicel-derived reference sample. Most importantly, the HPLC measurements proved a sufficiently high glucose purity ( $\sim 90 \%$, Table S3 in ESI $\dagger$ ) for cellulose triacetate obtained from pretreated bagasse. This glucose concentration reached the value for cellulose triacetate from Avicel. Overall, the simple synthesis of enriched cellulose triacetate was achieved by the combination of the mild acid-pretreatment of bagasse, direct transesterification in EmimOAc/DMSO, and subsequent reprecipitation in $\mathrm{MeOH}$.

\section{Conclusions}

The bifunctional use of EmimOAc enabled the homogeneous transesterification of lignocellulose without any corrosive 
reagents and additional catalysts. The reaction medium from the transesterification of bagasse was subjected to a simple reprecipitation in $\mathrm{MeOH}$, essentially fractionating into polysaccharide acetate and lignin acetate. In addition, the mild acid-pretreated bagasse was employed as the starting biomass to realise the facile enrichment of cellulose triacetate with a high glucose purity $(\sim 90 \%)$ without using a harsh delignification process. Therefore, we believe that the reported protocol can provide a direct path toward the production of important polymeric materials from biomass.

\section{Conflicts of interest}

The authors declare no competing financial interest.

\section{Acknowledgements}

This study was promoted by the Center of Innovation Science and Technology based Radical Innovation and Entrepreneurship Program (COI stream) of the Science and Technology Agency of Japan (JST).

\section{Notes and references}

1 D. Klemm, B. Heublein, H.-P. Fink and A. Bohn, Angew. Chem., Int. Ed., 2005, 44, 3358-3393.

2 G. Chatel, K. De Oliveira Vigier and F. Jerome, ChemSusChem, 2014, 7, 2774-2787.

3 F. Shafizadeh, Pure Appl. Chem., 1973, 35, 195-208.

4 M. Ionita, L. E. Crica, S. I. Voicu, A. M. Pandele and H. Iovu, Polym. Adv. Technol., 2016, 27, 350-357.

5 X. Fan, Z.-W. Liu, J. Lu and Z.-T. Liu, Ind. Eng. Chem. Res., 2009, 48, 6212-6215.

6 D. Hoffmann, I. Hoffmann and K. El-Bayoumy, Chem. Res. Toxicol., 2001, 14, 767-790.

7 T. C. Nirmale, I. Karbhal, R. S. Kalubarme, M. V. Shelke, A. J. Varma and B. B. Kale, ACS Appl. Mater. Interfaces, 2017, 9, 34773-34782.

8 H. Soeta, S. Fujisawa, T. Saito, L. Berglund and A. Isogai, ACS Appl. Mater. Interfaces, 2015, 7, 11041-11046.

9 K. Jedvert and T. Heinze, J. Polym. Eng., 2017, 37, 845-860. 10 S. Liu and K. J. Edgar, Carbohydr. Polym., 2017, 162, 1-9.

11 S. Tanaka, T. Iwata and M. Iji, ACS Sustainable Chem. Eng., 2017, 5, 1485-1493.

12 R. A. Sheldon, Green Chem., 2014, 16, 950-963.

13 K. J. Edgar, C. M. Buchanan, J. S. Debenham, P. A. Rundquist, B. D. Seiler, M. C. Shelton and D. Tindall, Prog. Polym. Sci., 2001, 26, 1605-1688.

14 W. O. S. Doherty, P. Mousavioun and C. M. Fellows, Ind. Crops Prod., 2011, 33, 259-276.

15 R. Singh, A. Shukla, S. Tiwari and M. Srivastava, Renewable Sustainable Energy Rev., 2014, 32, 713-728.

16 M. Alekhina, O. Ershova, A. Ebert, S. Heikkinen and H. Sixta, Ind. Crops Prod., 2015, 66, 220-228.

17 T. Q. To, C. Kenny, S. Cheong and L. Aldous, Green Chem., 2017, 19, 2129-2134.
18 J. S. Kim, Y. Y. Lee and T. H. Kim, Bioresour. Technol., 2016, 199, 42-48.

19 S. Laurichesse and L. Avérous, Prog. Polym. Sci., 2014, 39, 1266-1290.

20 F. S. Chakar and A. J. Ragauskas, Ind. Crops Prod., 2004, 20, 131-141.

21 T. Welton, Chem. Rev., 1999, 99, 2071-2083.

22 W. L. Hough and R. D. Rogers, Bull. Chem. Soc. Jpn., 2007, 80, 2262-2269.

23 R. P. Swatloski, S. K. Spear, J. D. Holbrey and R. D. Rogers, J. Am. Chem. Soc., 2002, 124, 4974-4975.

24 C. Achtel, K. Jedvert, B. Kosan, O. A. El Seoud and T. Heinze, Macromol. Chem. Phys., 2017, 218, 1700208.

25 T. Kakko, A. W. T. King and I. Kilpelainen, Cellulose, 2017, 24, 5341-5354.

26 H. Hanabusa, E. I. Izgorodina, S. Suzuki, Y. Takeoka, M. Rikukawa and M. Yoshizawa-Fujita, Green Chem., 2018, 20, 1412-1422.

27 R. Kakuchi, M. Yamaguchi, T. Endo, Y. Shibata, K. Ninomiya, T. Ikai, K. Maeda and K. Takahashi, $R S C$ Adv., 2015, 5, 72071-72074.

28 R. Kakuchi, R. Ito, S. Nomura, H. Abroshan, K. Ninomiya, T. Ikai, K. Maeda, H. J. Kim and K. Takahashi, RSC Adv., 2017, 7, 9423-9430.

29 Q. V. Nguyen, S. Nomura, R. Hoshino, K. Ninomiya, K. Takada, R. Kakuchi and K. Takahashi, Polym. J., 2017, 49, 783-787.

30 C. S. Lovell, A. Walker, R. A. Damion, A. Radhi, S. F. Tanner, T. Budtova and M. E. Ries, Biomacromolecules, 2010, 11, 2927-2935.

31 S. Koehler, T. Liebert, M. Schoebitz, J. Schaller, F. Meister, W. Guenther and T. Heinze, Macromol. Rapid Commun., 2007, 28, 2311-2317.

32 I. Kilpelaeinen, H. Xie, A. King, M. Granstrom, S. Heikkinen and D. S. Argyropoulos, J. Agric. Food Chem., 2007, 55, 91429148.

33 N. Sun, M. Rahman, Y. Qin, M. L. Maxim, H. Rodriguez and R. D. Rogers, Green Chem., 2009, 11, 646-655.

34 M.-J. Chen, R.-M. Li, X.-Q. Zhang, J. Feng, J. Feng, C.-F. Liu and Q.-S. Shi, ACS Sustainable Chem. Eng., 2017, 5, 360-366.

35 B. Adney and J. Baker, Laboratory analytical procedure, 1996, 6, 1-8.

36 Y. Zhao, X. Liu, J. Wang and S. Zhang, J. Phys. Chem. B, 2013, 117, 9042-9049.

37 J.-M. Andanson, E. Bordes, J. Devemy, F. Leroux, A. A. H. Padua and M. F. C. Gomes, Green Chem., 2014, 16, 2528-2538.

38 C. Li, B. Knierim, C. Manisseri, R. Arora, H. V. Scheller, M. Auer, K. P. Vogel, B. A. Simmons and S. Singh, Bioresour. Technol., 2010, 101, 4900-4906.

39 S. Hama, K. Nakano, K. Onodera, M. Nakamura, H. Noda and A. Kondo, Bioresour. Technol., 2014, 157, 1-5.

40 A. Sluiter, B. Hames, R. Ruiz, C. Scarlata, J. Sluiter, D. Templeton and D. Crocker, Laboratory analytical procedure, 2008, 1617, 1-16. 
41 H. Sakai, K. Kuroda, S. Muroyama, T. Tsukegi, R. Kakuchi, K. Takada, A. Hata, R. Kojima, T. Ogoshi, M. Omichi, K. Ninomiya and K. Takahashi, Polym. J., 2018, 50, 281-284. 42 A. Granata and D. S. Argyropoulos, J. Agric. Food Chem., 1995, 43, 1538-1544.
43 Y. Pu, S. Cao and A. J. Ragauskas, Energy Environ. Sci., 2011, 4, 3154-3166.

44 Fed. Regist., 2002, 67, 70835-70839.

45 S. Gillet, M. Aguedo, L. Petitjean, A. R. C. Morais, A. M. da Costa Lopes, R. M. Lukasik and P. T. Anastas, Green Chem., 2017, 19, 4200-4233. 